\title{
Type 1 diabetes mellitus and major depressive disorder: evidence for a biological link
}

\author{
D. J. Korczak • S. Pereira • K. Koulajian • A. Matejcek • \\ A. Giacca
}

Received: 17 February 2011 / Accepted: 27 May 2011 / Published online: 26 July 2011

(C) Springer-Verlag 2011

\begin{abstract}
Aims/hypothesis A growing body of research suggests that the prevalence of major depressive disorder (MDD) in children and youth with type 1 diabetes mellitus is significantly higher than that of youth without type 1 diabetes and is associated with increased illness severity. The objective of this article is to review the current literature on the pathophysiology of these two common diseases with respect to potential areas of overlapping biological dysfunction.

Methods A search of English language articles published between 1966 and 2010 was conducted and augmented with manual review of reference lists from the identified publications. Results The evidence suggests plausible mechanisms whereby a biological relationship between type 1 diabetes and MDD may exist. These include the effects of circulating cytokines associated with autoimmune diabetes, the direct impact of insulin deficiency on neurogenesis/neurotransmitter metabolism, the effects of the chronic hyperglycaemic state, occur-
\end{abstract}

\section{J. Korczak $(\bowtie)$}

Department of Psychiatry, The Hospital for Sick Children,

Rm 1145 Burton Wing, 555 University Avenue,

Toronto, ON, Canada M5G 1X8

e-mail: daphne.korczak@sickkids.ca

S. Pereira $\cdot$ K. Koulajian $\cdot$ A. Giacca

Department of Physiology, University of Toronto,

Toronto, ON, Canada

A. Matejcek

Department of Medicine, University of Ottawa,

Ottawa, ON, Canada

\section{A. Giacca}

Department of Medicine, University of Toronto,

Toronto, ON, Canada

A. Giacca

Institute of Medical Sciences, University of Toronto,

Toronto, ON, Canada rence of iatrogenic hypoglycaemia and the impact of basal hyperactivity of the hypothalamic-pituitary-adrenal axis.

Conclusions/interpretation Shared biological vulnerabilities may be implicated in the comorbidity of type 1 diabetes and MDD. Further research is warranted to determine the magnitude of associations and confirm their observation in clinical populations.

Keywords Children and adolescents - Cytokines .

Hypothalamic-pituitary-adrenal axis · Inflammation · Major depressive disorder - Oxidative stress · Pathophysiology.

Psychology Review Type 1 diabetes mellitus

$\begin{array}{ll}\text { Abbreviations } \\ \text { ACTH } & \text { Adrenocorticotropic hormone } \\ \text { BCAA } & \text { Branched chain amino acid } \\ \text { BDNF } & \text { Brain-derived neurotrophic factor } \\ \text { CNS } & \text { Central nervous system } \\ \text { DST } & \text { Dexamethasone suppression test } \\ \text { HPA } & \text { Hypothalamic-pituitary-adrenal } \\ \text { LNAA } & \text { Large neutral amino acid } \\ \text { MDD } & \text { Major depressive disorder } \\ \text { ROS } & \text { Reactive oxygen species } \\ \text { sICAM } & \text { Soluble intracellular adhesion molecule } \\ \text { SOD } & \text { Superoxide dismutase } \\ \text { STZ } & \text { Streptozotocin }\end{array}$

Major depressive disorder (MDD) is highly prevalent among children and adolescents with type 1 diabetes mellitus. The prevalence of MDD among youth with type 1 diabetes (20$27 \%$ ) is at least two to three times greater than the 5-8\% background rate of MDD reported for non-diabetic youth [1, 2]. Early-onset MDD is severe, and in combination with diabetes (the third most common chronic disease in childhood), is associated with poorer diabetes control, increased 
diabetes-related complications, increased frequency of emergency department visitation [3] and hospitalisations [4], greater functional impairment, increased suicidality [5] and higher healthcare costs [6]. Our current understanding of poor diabetes control in adolescents with MDD is based primarily on an indirect relationship between the two illnesses in which the neurovegetative symptoms and negative self-cognitions of MDD lead to non-adherence to the insulin regimen. As a result, current approaches to poorly controlled type 1 diabetes centre on individual and family therapy, which on the whole have yielded suboptimal improvements in diabetes control [7]. However, growing independent interest in the pathophysiology of MDD and type 1 diabetes has demonstrated exciting biological parallels in structural brain abnormalities, neurocognitive symptoms and neuroendocrine dysfunction common to both disease states [8]. These independent findings, and not only the effect of MDD on treatment compliance, are consistent with research reporting that adolescents with poorly controlled type 1 diabetes exhibit a greater burden of MDD than those with well-controlled diabetes $[3,9,10]$. Taken together, these data suggest that closer examination of areas of pathophysiological overlap and the potential for bidirectional effects of MDD and type 1 diabetes warrant further exploration. To date, reviewers examining the comorbidity of MDD and diabetes mellitus have largely focused on type 2 diabetes $[8$, 11], which occurs primarily in adulthood. Present reviews aimed at understanding the co-occurrence of MDD and diabetes mellitus insufficiently address clinical data demonstrating increased rates of MDD in studies of children and adolescents with diabetes. As such, the literature concerning those with potentially the longest duration of comorbid illness remains sparse. The purpose of this investigation is to review the present knowledge with respect to the pathophysiological basis of depression and type 1 diabetes, with the goal of elucidating areas of shared vulnerabilities to further the current understanding of the increased prevalence rate of MDD in children and adolescents with type 1 diabetes.

MEDLINE and PubMed searches of English language articles published between 1966 and 2010 were conducted using the search terms 'diabetes mellitus', 'type 1 diabetes', 'depression', 'brain', cross-referenced with 'inflammation', 'cytokines', 'oxidative stress', 'antioxidant enzyme', 'cortisol', 'glucocorticoid', 'hypothalamic-pituitary-adrenal axis', 'dexamethasone', 'hyperglycaemia', 'hypoglycaemia', 'neurobiology' or 'cognitive function' and augmented with manual review of reference lists. An additional search of English language articles published between 1966 and 2011 using the search terms 'type 1 diabetes', 'diabetes mellitus', 'depression', crossreferenced with 'brain-derived neurotrophic factor', 'vitamin D' or 'polyunsaturated fatty acids' was conducted. Using this strategy, 1,441 articles were identified (Table 1). Articles selected for review were not limited to human studies and were based on adequacy of sample size, the use of standardised experimental procedures, validated assessment measures and overall manuscript quality. Assessment of article suitability was assigned to study authors based on their content expertise.

In this review the literature is discussed in the following categories: (1) immuno-inflammatory factors, including cytokine activation and oxidative stress; (2) endocrinological factors, including insulin and glucose dysregulation and cortisol hypersecretion; and (3) neurobiological abnormalities, including structural, functional and cognitive findings.

\section{Immuno-inflammatory factors}

Numerous inflammatory and immunological mediators have been implicated in the pathology of both type 1 diabetes and MDD; the most prominent of these pertain to the roles of cytokines and oxidative stress in producing and perpetuating these diseases.

Cytokines Type 1 diabetes occurs as a result of autoimmune destruction of the insulin-producing beta cells of the islets of Langerhans in the pancreas. The specific aetiology and pathogenesis of the disease is unclear. However, a prolonged insulitis phase, whereby leucocytes invade and damage the beta cells, may be covertly present for years prior to progression to frank diabetes. Populations at risk for the development of type 1 diabetes (i.e. those presumed to have insulitis) show elevated cytokine secretion by circulating cells [12]. In patients with type 1 diabetes, anti-islet antibody positivity can be predicted by the circulating cytokine profile. This suggests that circulating cytokines are related to beta cell immune responses

Table 1 Literature search terms used and articles identified

\begin{tabular}{ll}
\hline Search terms & $\begin{array}{l}\text { Number of articles } \\
\text { initially identified }\end{array}$ \\
\hline $\begin{array}{l}\text { Diabetes mellitus, type 1 diabetes, depression, brain, cross-referenced with inflammation, } \\
\text { cytokines, oxidative stress, antioxidant enzyme, cortisol, glucocorticoid, }\end{array}$ & 892 \\
$\begin{array}{l}\text { hypothalamic-pituitary-adrenal axis, dexamethasone, hyperglycaemia, } \\
\text { Hypoglycaemia, neurobiology, or cognitive function cross-referenced with type 1 diabetes, depression }\end{array}$ & 492 \\
$\begin{array}{l}\text { Type } 1 \text { diabetes, diabetes mellitus, depression, cross-referenced with brain-derived } \\
\text { neurotrophic factor, vitamin D or polyunsaturated fatty acids }\end{array}$ & 64 \\
\hline
\end{tabular}


[13]. Clinical studies show that the pathogenesis and clinical characteristics of type 1 diabetes differ depending on the age at onset. A younger age at onset is associated with more sudden and extensive beta cell destruction $[14,15]$, and is thus related to a greater but less persistent inflammatory response as continuation of the immuno-inflammatory response in type 1 diabetes depends upon the existence of beta cells, which act as a source of antigen. The effect of the magnitude and duration (i.e. acute and extensive vs prolonged and moderate) of beta cell destruction on the level of circulating cytokines, however, remains unclear [16]. Because of the effects of cytokines on the central nervous system (CNS; discussed below), a link between type 1 diabetes and MDD can be postulated based on the levels of circulating cytokines.

In addition to the inflammation resulting from beta cell destruction, clinical studies show that hyperglycaemia in itself augments cytokine production. Patients with poorly controlled type 1 diabetes show increased plasma IL-4 and IL- 6 concentrations and increased production of TNF $\alpha$ $(p=0.01)$ and IL-10 $(p=0.007)$ by circulating leucocytes [17]. In addition, patients with type 1 diabetes may be more susceptible than controls to an elevation in plasma TNF $\alpha$ in response to acute hyperglycaemia [18].

Some, but not all, studies have similarly shown increased immune activity in depression [19]. Data from the Third National Health and Nutrition Examination Survey, for example, showed that a lifetime history of major depression was associated with a $64 \%$ increased risk of having elevated C-reactive protein levels (OR 1.64; 95\% CI 1.2, 2.2) [20]. Other pro-inflammatory cytokines reported to be increased in MDD include IL-6, IL-1, TNF $\alpha$ and IFN $\alpha$ [21-23]. Of note, IL-6, IL-1 and TNF $\alpha$ have been shown to induce a constellation of non-specific symptoms, including fatigue, anorexia, decreased psychomotor activity, sleep disturbance and decreased self-care, collectively referred to as 'sickness behaviour' and reminiscent of depressive symptoms [24]. Although these findings are of particular salience given the pathophysiology of type 1 diabetes described above, they are the result of studies of adult populations. In these adult populations, however, evidence does support the role of proinflammatory cytokines as mediators in the potential interaction between depression and diabetes, in part via cytokine activation of the hypothalamic-pituitary-adrenal (HPA) axis [25]. Whether levels of pro-inflammatory cytokines are also increased in child and adolescent populations with MDD remains unknown. Studies of inflammatory mediators in children and adolescents with type 1 diabetes are needed to further delineate their potential contribution to the preexistence or development of MDD in this population.

Increased circulating cytokines are also associated with increased circulating cellular adhesion molecules, particularly soluble intracellular adhesion molecule 1 [sICAM-1]. Elevated sICAM-1 concentrations are associated with depression in adult patients with cardiovascular disease (OR 1.22; 95\% CI 1.02, 1.46) [26] and have been hypothesised to mediate the relationship between depression and other metabolic disorders, including diabetes [27]. Further investigation is needed to assess whether an elevation of cellular adhesion molecules is similarly found in paediatric patients and whether this increase is of specific relevance to the pathogenesis of depression or simply a reflection of a persistent level of chronic inflammation.

Early literature reporting a decreased dietary intake of $n-3$ polyunsaturated fatty acids in individuals with MDD, coupled with evidence of the anti-inflammatory properties of these substances, generated interest in the potential use of these agents as adjuvant treatments for depression among patients with diabetes. Recently, however, epidemiological studies examining the association between low levels of $n-3$ polyunsaturated fatty acids and depression have shown mixed results $[28,29]$, and treatment studies have not confirmed additional benefits of polyunsaturated fatty acids in the treatment of depression in the presence of either type 1 or type 2 diabetes or other inflammatory diseases [30, 31]. Moreover, in a recent prospective study, neither dietary intake of $n-3$ fatty acids nor erythrocyte membrane $n-3$ fatty acid levels were associated with conversion to type 1 diabetes among high-risk children [32]. However, adequate assessment of the potential role of $n-3$ polyunsaturated fatty acid deficiency in the co-occurrence of depression and type 1 diabetes requires further study in larger samples and among comorbid youth. Similarly, investigators have suggested a possible role for vitamin D insufficiency/deficiency in the pathogenesis of type 1 diabetes [33], type 2 diabetes [34] and MDD [35]. However, research examining these associations has been subject to numerous methodological limitations [36, 37] that obscure clear interpretation of these data and underscore the need for further study of the potential contribution of vitamin $\mathrm{D}$ insufficiency to depression and/or diabetes.

Oxidative stress Oxidative stress has emerged as a potential mechanism underlying cellular dysfunction in many pathological processes, including those leading to diabetes and mood disorders [38-40]. An overproduction of reactive oxygen species (ROS) can overwhelm antioxidant defences and result in oxidative damage, including protein oxidation, lipid peroxidation and DNA damage, which may lead to cell death [41]. The beta cells of the pancreas and the cells of the CNS are both particularly vulnerable to the effects of oxidative stress, owing to their low levels of antioxidant enzyme activity compared with other tissues [42, 43].

A recent study of 176 children with type 1 diabetes demonstrated increased markers of oxidative stress in plasma and circulating cells, including diminished glutathione peroxidase activity $(p<0.0001)$ and increased protein carbonyl $(p<0.0001)$ and lipid peroxidation $(p<0.0001)$ 
levels, compared with those in 140 age-, sex- and BMImatched healthy controls [44]. Improved glycaemic control has been shown to diminish markers of macromolecular oxidation and improve glutathione levels in patients with type 1 diabetes, indicating that hyperglycaemia can induce oxidative stress in type 1 diabetes [44, 45].

Clinical studies of paediatric patients with diabetes support these findings. Decreased antioxidative protection from lipid peroxidation and nitrate/nitrite overproduction occurs in children with type 1 diabetes early in the disease course and increases with longer illness duration [46]. This is further supported by studies of rodent models of type 1 diabetes, such as streptozotocin (STZ)-treated rodents. STZ-treated rats show the following markers of oxidative stress: increased activity of ROSproducing enzymes and blunted antioxidant defences in various regions of the brain. Furthermore, in these models the level of oxidative stress within the brain is decreased by administration of antioxidants such as vitamin E, $N$-acetylcysteine, and resveratrol [47-49]. The mechanism of hyperglycaemia-induced oxidative stress may involve mitochondrial ROS (superoxide) generation, which is elevated in hyperglycaemic conditions as a result of elevated oxidative phosphorylation $[50,51]$. In addition, hyperglycaemia results in increased levels of cytokines [52], which generate ROS in part by stimulating transcription of xanthine oxidase [53], the activity of which is elevated in various brain regions of STZ-treated rats [49].

The CNS has been reported to be at particular risk of ROS-induced harm owing to its high level of oxidative metabolic activity and high polyunsaturated fat content. Clinical studies have found depression to be characterised by elevated markers of oxidative stress, such as serum lipid peroxidation [54], and diminished antioxidant levels, such as serum vitamin $\mathrm{E}$ [55] and plasma vitamin C [54]. Correlations between increasing depression severity and the magnitude of superoxide dismutase (SOD) activity have also been reported, such that patients with severe MDD have been found to have greater SOD activity than those experiencing mild $(p<0.001)$ or moderate $(p<0.001)$ disease [56]. Moreover, post-mortem analyses indicate that SOD protein levels are augmented in the prefrontal cortex of patients with depression, which may suggest compensation for oxidative stress in brain tissue in this patient population [57]. Hypercortisolism, which is present in depression (and diabetes), as described below, can also contribute to increased oxidative stress $[58,59]$. In rodent studies, experimental models of depression (chronic mild stress model) demonstrate that glucocorticoid hypersecretion causes oxidative stress, both by increasing lipid peroxidation in the brain cortex and medulla and by decreasing glutathione levels in the medulla [60]; this finding requires confirmation in humans. Furthermore, antidepressant treatment in humans and animals has been shown to alleviate oxidative stress $[54,60]$. The mechanism by which oxidative stress may cause depression is not known; however, one potential area of investigation is oxidative stress-induced mitochondrial dysfunction, which is characteristic of depression [61] and may induce altered neuronal activity or death [61].

Studies investigating the link between diabetes and depressive-like behaviour in murine models have yielded interesting results. STZ-treated rats demonstrate increased ROS and decreased total antioxidant reactivity compared with non-diabetic rats when subjected to an experimental model of depression (forced swim test) [62]. Both ROS levels and total antioxidant reactivity improved towards non-diabetic values on administration of a drug that produces an antidepressant effect in these animals (clonazepam) [63, 64]. These findings are consistent with previous reports suggesting that the mitochondrial electron transport chain may be an overlooked target of antidepressant action [65]. Thus, the evidence to date suggests that oxidative stress may be a key element in the pathophysiology of comorbid type 1 diabetes and MDD, and this requires further investigation.

- Patients with type 1 diabetes show increased plasma levels of cytokines (IL-4, IL-6, IL-10 and TNF $\alpha$ ) as a result of beta cell destruction and hyperglycaemia. Patients with MDD show similar increases in immune activity.

- Increased plasma IL-6, IL-1 and TNF $\alpha$ have been shown to induce sickness behaviour, a constellation of symptoms associated with MDD.

- Pro-inflammatory cytokines may mediate the relationship between type 1 diabetes and depression via activation of the HPA axis.

- Elevated markers of oxidative stress occur in both patients with type 1 diabetes and in those with MDD. Antidepressant treatment has been shown to reduce oxidative stress in rodent models of diabetes.

\section{Endocrinological factors}

A direct link between type 1 diabetes and MDD is provided by the hallmarks of diabetes: abnormalities in insulin and glucose concentrations, including insulin deficiency, hyperglycaemia and iatrogenic hypoglycaemia. The role of hyperglycaemia as a generator of inflammation/oxidative stress has been described above. In this section the role of insulin deficiency and insulin-induced hypoglycaemia will be reviewed, together with the indirect link between type 1 diabetes and MDD provided by the diabetes-induced activation of the HPA axis. 
Insulin deficiency and insulin-induced hypoglycaemia The complexity of the roles of insulin, insulin receptors, and insulin-sensitive glucose transporters in cognitive and emotional processes is increasingly being recognised. Insulin binding in the rodent brain has been found to be highest in those regions that subserve cognitive and emotional functions, including the hippocampus, hypothalamus, amygdala, cortex, olfactory bulb and septum [66]. Similarly, insulin-sensitive GLUT8 glucose transporters are selectively distributed in the hippocampus and hypothalamus [66], suggesting that glucose uptake is insulin-stimulated in these regions; challenging earlier presumptions of the brain as an 'insulin-insensitive' organ.

There is now evidence that insulin exerts important functions in neural development and synaptic plasticity [67]. Systemic or intranasal administration of insulin enhances learning and memory and has been proposed as a treatment for Alzheimer's disease [68]. These findings have led to the hypothesis that insulin insufficiency may lead to the defects in neurocognition commonly observed in MDD (discussed below).

In addition, a lack of insulin may lead to symptoms of MDD secondary to abnormal amino acid metabolism. The production of serotonin, a key neurotransmitter associated with MDD, in the brain depends on the availability and transport of its amino acid precursor, tryptophan [69]. In the plasma, tryptophan competes with other large neutral amino acids (LNAAs), such as the branched chain amino acids (BCAAs; leucine, isoleucine, and valine) for transport across the brain-blood barrier, as they use the same carrier [70]. Hence, the ratio of plasma tryptophan to total LNAAs is used as a marker of tryptophan availability in the brain [71, 72]. In healthy individuals, insulin diminishes the concentration of plasma BCAAs [73] because of insulin inhibition of protein breakdown [74]. Patients with type 1 diabetes show elevated concentrations of plasma BCAAs [72, 75] and markers of decreased brain serotonin system function [76]. Furthermore, STZ-treated rats have increased plasma BCAA concentrations, plus low levels of tryptophan and diminished serotonin production in the brain $[69,71]$. Insulin treatment increases brain levels of tryptophan, and thereby the substrate for serotonin production, in these animals [71, 77].

Conversely, an excess of insulin during type 1 diabetes treatment and the resultant CNS effects of profound hypoglycaemia may also be associated with the neurocognitive deficits common to patients with early-onset type 1 diabetes and MDD. Individuals with type 1 diabetes are thought to be at risk of cognitive impairment as a result of repeated episodes of hypoglycaemia [78], although studies examining the effects of hypoglycaemia on cognitive function have produced discordant results $[79,80]$. This discrepancy may relate to differences in the age of diabetes onset of study participants, as younger age at diagnosis ( $<5$ years) has been associated with poorer cognitive outcomes, including poorer attention and school achievement $(F=2.66, p<0.01)$, decreased visuospatial ability $(F=3.874, p<0.001)$, greater difficulties with learning and memory skills $(F=2.34, p<0.01)$, and poorer global intelligence scores $(F=2.13, p=0.02)$ as compared with older age at onset [81]. Another explanation for the disagreement between reports is that individuals with more hypoglycaemia may have tighter glycaemic control overall (i. e. shorter duration of chronic hyperglycaemia, and thus less oxidative stress/inflammation), which may compensate for the neurocognitive damage from hypoglycaemia [82]. Clinically, this pattern of neurocognitive deficit overlaps with those commonly experienced in MDD (discussed below). In addition to producing a depressive neurocognitive profile, hypoglycaemic events have also been associated with elevated levels of depressive symptoms [83].

Hyperactivity of the HPA axis One putative link between type 1 diabetes and MDD is basal hyperactivity of the HPA axis, which is common to both diseases. Diabetes is associated with a hypercortisolaemic state, characterised by elevated levels of circulating cortisol and increased $24 \mathrm{~h}$ levels of urinary free cortisol [84]. Disrupted control of adrenocorticotropic hormone (ACTH) release from pituitary corticotrophs and direct stimulation of corticotropin-releasing hormone of the adrenal gland with or without the release of ACTH leads to hyperactivation of the HPA in patients with diabetes [85]. Furthermore, the impairment of glucocorticoid-negative feedback sensitivity in patients with diabetes also results in increased activity of the HPA axis: following glucocorticoid administration, these patients exhibit a greater incidence of nonsuppression of pituitary-adrenal activity compared with non-diabetic individuals $(43 \%$ vs $7 \%$, respectively; $p<0.01)$ [86]. Studies in STZ-treated rats have also shown that the basal hyperactivation of the HPA axis is associated with further decreased responsiveness of the HPA axis to stress, for example, to insulin-induced hypoglycaemia. While basal hyperactivity is secondary to the lack of insulin in STZ induced-diabetes, the reduced response to hypoglycaemia is due to the chronic hyperglycaemic state [87].

The functional abnormalities of the HPA axis in the depressed state are well described and include increased plasma cortisol concentrations [88], an enlarged adrenal cortex [89] and elevated concentrations of cortisol and corticotropin-releasing factor in the cerebrospinal fluid [90]. Cortisol levels are not suppressed following dexamethasone administration (dexamethasone suppression test [DST]) in depressed patients and this non-suppression is resolved with treatment of the psychiatric disorder. Furthermore, a meta-analysis of 14 studies by Ribeiro et al. has shown that 
normalisation of the response to the DST precedes clinical recovery in patients with MDD, with persistence of nonsuppression portending poorly for clinical outcome (normalisation: $63 \%$ in patients with good outcome vs $31 \%$ in patients with poor outcome; $p<0.01$ ) [91]. Additionally, a link between elevated cortisol levels and mood alterations is suggested by investigations of patients with primary hypercortisolism caused by Cushing's syndrome. The syndrome is associated with symptoms similar to those of depression, including depressed or labile mood, fatigue, insomnia, poor concentration and decreased energy [92]. Patients with Cushing's syndrome have an increased incidence of depressive illness, with rates as high as $67 \%$ reported [93]. The depressive symptoms in Cushing's syndrome are reported to resolve with treatment of the hypercortisolaemia [93]. Sustained exposure to supraphysiological levels of glucocorticoid decreases local cerebral glucose utilisation and inhibits glucose uptake in hippocampal neurons in vitro [94]. Corticosteroids have been demonstrated to exert tonic inhibitory control of hippocampal 5- $\mathrm{HT}_{1 \mathrm{~A}}$ receptors [95]; this finding is of particular salience given the serotonin deficiency that occurs in depression [96]. Taken together, these findings have led to the hypothesis of an aetiological role for hypercortisolaemia in the pathophysiology of depression and to suggestions of new treatment approaches to depression aimed at directly targeting HPA axis abnormalities [97]. However, it is also possible that MDD can lead to HPA abnormalities because of the non-specific stress of depression. In addition, a common heritable basis between HPA dysfunction and MDD has been suggested based on the finding that non-depressed first-degree relatives of depressed patients exhibit DST abnormalities as a trait [98]. Once established, the HPA axis dysregulation and hypercortisolaemia may further contribute to a hyperglycaemic or poorly controlled diabetic state and has been associated with increased chronic complications of diabetes in adult studies [99].

- Insulin binding in the brain is highest in areas that subserve cognitive and emotional functions.

- Lack of insulin leads to abnormal amino acid metabolism, which is associated with decreased brain serotonin production.

- Insulin-induced hypoglycaemia may be associated with the neurocognitive deficits seen in patients with early-onset type 1 diabetes and may be a risk factor for the development of MDD.

- Hyperactivity of the HPA axis has been noted in both type 1 diabetes and MDD and provides a putative link between type 1 diabetes and MDD comorbidity.

\section{Neurobiological factors}

Whether the result of a lack of insulin, chronic hyperglycaemia, frequent hypoglycaemic events or hypercortisolism, neurocognitive deficits have been described in diabetes. A recent meta-analysis of 33 studies by Brands et al. [100] found that individuals with type 1 diabetes demonstrate reduced overall cognition $(d=-0.7)$, decreased speed of information processing $(d=-0.3)$, decreased attention $(d=-0.3)$ and reduced psychomotor efficiency $(d=-0.6)$ compared with non-diabetic controls. These abnormalities are consistent with those commonly found in patients with MDD [101].

The CNS structures implicated in the neurocognitive deficits described in studies of individuals with diabetes are similar to those identified in the pathology of MDD. For example, the hippocampus has been described as a region of interest in diabetes [102]. This is in part due to its high density of insulin receptors and its insulin-sensitive glucose transporters (discussed above) [66]. In STZ-diabetic rats, hypercortisolism has been implicated in neurodegeneration by way of persistently elevated glucocorticoid levels leading to neurodegeneration or suppressed neurogenesis of the CA3 pyramidal neurons of the hippocampus [103]. Decreased hippocampal volumes have been reported in patients with type 2 diabetes and in non-diabetic individuals with abnormal glucose tolerance [8]. The hippocampus has also been implicated in neurocognitive dysfunction in children and adolescents with type 1 diabetes, particularly in those with a younger age of type 1 diabetes onset. Ferguson et al. [104] found an increased number of hippocampal small punctuate white matter lesions in those with early-onset diabetes (onset younger than 7 years of age). This finding is consistent with a recent study by Ho et al. [105] that identified mesial temporal lobe sclerosis more frequently in individuals with early-onset vs later onset of type 1 diabetes. The sole study of hippocampal volumes in type 1 diabetic patients did not find a difference in hippocampal size in 13 subjects with and without type 1 diabetes [106]; however, this small study excluded individuals with current depression or a history of depression and in doing so may have inadvertently omitted those with a hippocampal pathology, as discussed below. Whether the neuroimaging findings in early-onset vs later onset type 1 diabetes in youth are specific to age at onset or are secondary to illness duration is unclear. Other neuroimaging findings in individuals with diabetes have reported cerebral atrophy [107] and reduced cortical cerebral blood flow and glucose metabolism [108] in type 1 diabetic patients compared with controls.

The functional and structural CNS abnormalities described in MDD are similar to those described in diabetes. Research examining CNS abnormalities in 
MDD has confirmed dysfunction of the hippocampus (memory, learning and control of autonomic and vegetative functions), hypothalamus (sleep and appetite regulation, sexual dysfunction), amygdala (anxiety, fear and establishment of emotional valence of memory) and, more recently, of the nucleus accumbens (pleasure) in affected individuals [109]. Studies have established a central role of the hippocampus in MDD, noting a decrease in hippocampal volume in affected individuals [110], and have postulated that a deficit of neurotrophic support may contribute to the hippocampal pathology (discussed below) [109]. Stress-associated hypercortisolism may be involved in hippocampal dysfunction, as sustained high levels of glucocorticoids have been shown to have adverse effects on hippocampal neurons, with decreased dendritic branching and impairment of neurogenesis reported [111]. In addition to reduced hippocampal volume, neuroimaging studies of individuals with MDD have revealed atrophy of the prefrontal cortex and biphasic changes of the amygdala based on illness chronicity [112]. These findings correlate with those of studies of cerebral perfusion and glucose metabolism in individuals with MDD, which demonstrated both a decrease in these factors in the prefrontal cortex and an increase in the amygdala in affected individuals [113]. These findings are reminiscent of those reported in type 1 diabetes above.

Brain-derived neurotrophic factor Recent evidence suggests a potential role for brain-derived neurotrophic factor (BDNF) in the relationship between depression and diabetes. High levels of BDNF are found in hippocampal neurons and these are decreased in adult depressed patients [109] but increase with improvements in depressive symptoms, duration of treatment and antidepressant use [114, 115]. Research confirming the associations of increased BDNF levels with neuronal survival and decreased BDNF levels with decreases in synaptic plasticity and neuronal atrophy has strengthened the evidence in favour of the neurotropic hypothesis of depression. This hypothesis postulates that growth factors act to transduce stressors into decreased rates of adult hippocampal neurogenesis, atrophic changes and impaired synaptic plasticity of hippocampal neurons, which may explain the cognitive impairment and hippocampal atrophy of depression [116]. However, findings of decreased hippocampal size in individuals with MDD appear to be associated with increased length of illness and are not present early in the course of MDD. In addition, BDNF levels decrease with age, highlighting the need for research in depressed youth.

Increasingly, BDNF has become recognised as a metabotrophic factor [117], with reduced levels reported in several cardiometabolic diseases, including type 2 diabetes. Studies investigating the relationship between BDNF and diabetes in adults demonstrate that BDNF levels are decreased in type 2 diabetes and are regulated in response to plasma levels of glucose [118]. In addition, studies of healthy adults have found that hyperglycaemia inhibits BDNF output from the brain [118]. Although models of obese diabetic mice have shown that BDNF exerts a protective effect on pancreatic islet cells [119], no studies have yet examined the role of BDNF in rodents or humans with type 1 diabetes

Taken together, these findings suggest that BDNF may play a role in both depression and type 1 diabetes; this factor requires examination in youth. The hyperglycaemic state of diabetes may inhibit hippocampal BDNF levels, resulting in MDD among vulnerable affected individuals. Determining the basis for this vulnerability is of key importance. Though nascent, the literature examining this topic suggests that early-life stress and BDNF genotype may interact to result in depression [120]. A recent study of 1,435 adults with a history of MDD reported that the experience of recent life stressors is associated with decreased levels of BDNF [121]. This is consistent with research on murine models of early-life stress, in which a depressed phenotype in rats experiencing early maternal separation is associated with a decrease in mossy fibre development and neurogenesis in the hippocampi of these animals [111].

- Striking similarities exist between the structural and functional abnormalities of the CNS described in MDD and those reported to occur in type 1 diabetes, including decreases in hippocampal volume, decreased cerebral perfusion and glucose metabolism, and similar patterns of neurocognitive deficits.

- Levels of BDNF are decreased in depression and increase in response to antidepressant treatment. BDNF output is inhibited by hyperglycaemia and is decreased in adults with type 2 diabetes.

\section{Conclusion}

The findings of research into the pathophysiology of type 1 diabetes and MDD suggest plausible mechanisms whereby a biological link between these illnesses may exist. These include the effects of circulating cytokines associated with autoimmune diabetes, the direct impact of insulin deficiency on neurogenesis/neurotransmitter metabolism, the effects of the chronic hyperglycaemic state, the occurrence of iatrogenic hypoglycaemia and the 


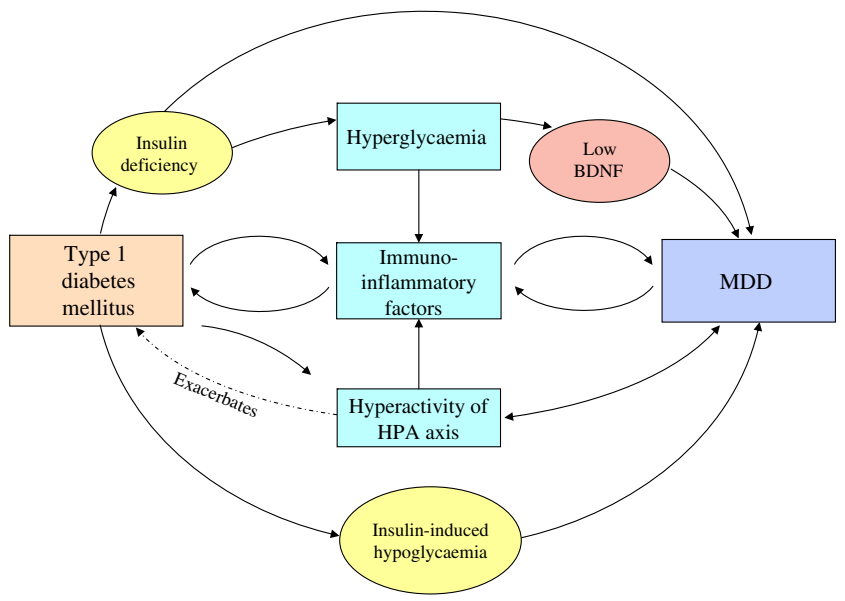

Fig. 1 The relationship between type 1 diabetes mellitus and MDD: possible pathways of association

impact of basal hyperactivity of the hypothalamicpituitary-adrenal axis (Fig. 1). Traditional conceptualisation of the increase in MDD prevalence among youth with type 1 diabetes has relied heavily on the psychological burden and non-specific stress of coping with a chronic illness, leading to nonadherence to the diabetes regimen. However, evidence suggests that the increase in MDD comorbidity among youth with type 1 diabetes might be due in part to the common pathophysiological processes that occur in these diseases. In addition, the biological correlates associated with the presence of MDD may be a barrier to achieving optimal diabetes control. Prospective longitudinal studies of children and adolescents with type 1 diabetes are limited, and research examining these pathways in comorbid clinical populations is lacking. Further investigation aimed at understanding the longterm outcomes of patients with type 1 diabetes are needed to clarify potential pathways of comorbidity and advance the current understanding of the basis of the increased prevalence of MDD reported among children and adolescents with type 1 diabetes.

Contribution statement The authors contributed to the manuscript in the following ways: DK was responsible for the conception and formulation of the review. She authored the initial draft of the paper and has been responsible for incorporating all of the coauthors contributions into a cohesive manuscript. She has been directly involved in the ongoing revision prior to publication and has approved the final version. SP has contributed to the literature search and review, and drafted the sections of the manuscript pertaining to oxidative stress, inflammation and amino acids in diabetes. She has reviewed the final version of the paper prior to publication. KK has contributed to the literature search and review, and drafted the sections of the manuscript pertaining to the HPA axis and neurocognitive findings in diabetes. He has reviewed the final version of the paper prior to publication. AM has contributed to the design of the manuscript and has drafted the summary textboxes. She has revised it critically to improve clarity, meaning, and accuracy of the context of the cited references and has reviewed and approved the version to be published. AG was heavily involved in the conceptual work and re-design of the initial draft. She rationalised the categories of discussion of the literature and suggested alternate explanations for analysis and interpretation of the literature. She revised each manuscript draft critically for intellectual content and was instrumental in the design and drafting of the visual representation of the findings (Fig. 1). AG has seen and approved the final version to be published.

Duality of interest The authors declare that there is no duality of interest associated with this manuscript.

\section{References}

Papers of particular interest have been noted as:

- Of importance

•• Of major importance

1. Grey M, Whittemore R, Tamborlane W (2002) Depression in type 1 diabetes in children: natural history and correlates. J Psychosom Res 53:907-911

2. •- Kovacs M, Goldston D, Obrosky DS, Bonar LK (1997) Psychiatric disorders in youths with IDDM: rates and risk factors. Diabetes Care 20:36-44. This is the longest longitudinal study of psychiatric illness in children with type 1 diabetes, using gold-standard measurement methods for determination of psychopathology

3. Lawrence JM, Standiford DA, Loots B et al (2006) Prevalence and correlates of depressed mood among youth with diabetes: the SEARCH for Diabetes in Youth study. Pediatrics 117:1348-1358

4. Stewart SM, Rao U, Emslie GJ, Klein D, White PC (2005) Depressive symptoms predict hospitalization for adolescents with type 1 diabetes mellitus. Pediatrics 115:1315-1319

5. Goldston DB, Kelley AE, Reboussin DM et al (1997) Suicidal ideation and behavior and noncompliance with the medical regimen among diabetic adolescents. J Am Acad Child Adolesc Psychiatry 36:1528-1536

6. Leichter SB, See Y (2005) Problems that extend visit time and cost in diabetes care: 1 . how depression may affect the efficacy and cost of care of diabetic patients. Clin Diabetes 23:53-54

7. Murphy HR, Rayman G, Skinner TC (2006) Psycho-educational interventions for children and young people with type 1 diabetes. Diabet Med 23:935-943

8. McIntyre RS, Soczynska JK, Konarski JZ et al (2007) Should depressive syndromes be reclassified as "Metabolic Syndrome Type II"? Ann Clin Psychiatry 19:257-264

9. Hassan K, Loar R, Anderson BJ, Heptulla RA (2006) The role of socioeconomic status, depression, quality of life, and glycemic control in type 1 diabetes mellitus. J Pediatr 149:526-531

10. Moussa MA, Alsaeid M, Abdella N, Refai TM, Al-Sheikh N, Gomez JE (2005) Social and psychological characteristics of Kuwaiti children and adolescents with type 1 diabetes. Soc Sci Med 60:1835-1844

11. Goldbacher EM, Matthews KA (2007) Are psychological characteristics related to risk of the metabolic syndrome? A review of the literature. Ann Behav Med 34:240-252

12. - Rapoport MJ, Bistritzer T, Aharoni D et al (2005) TH1/TH2 cytokine secretion of first degree relatives of T1DM patients. Cytokine 30:219-227. This study examines cytokine secretion by 
circulating cells of the family members of patients with type 1 diabetes

13. Hanifi-Moghaddam P, Schloot NC, Kappler S, Seissler J, Kolb H (2003) An association of autoantibody status and serum cytokine levels in type 1 diabetes. Diabetes 52:1137-1142

14. Decochez K, Tits J, Coolens JL et al (2000) High frequency of persisting or increasing islet-specific autoantibody levels after diagnosis of type 1 diabetes presenting before 40 years of age. The Belgian Diabetes Registry. Diabetes Care 23:838-844

15. Sabbah E, Savola K, Ebeling T et al (2000) Genetic, autoimmune, and clinical characteristics of childhood- and adult-onset type 1 diabetes. Diabetes Care 23:1326-1332

16. - Uno S, Imagawa A, Okita K et al. (2007) Macrophages and dendritic cells infiltrating islets with or without beta cells produce tumour necrosis factor-alpha in patients with recentonset type 1 diabetes. Diabetologia 50:596-601. This study reports on the presence of proinflammatory cytokines and immune cells in the islet cells of patients with type 1 diabetes

17. Foss-Freitas MC, Foss NT, Rassi DM, Donadi EA, Foss MC (2008) Evaluation of cytokine production from peripheral blood mononuclear cells of type 1 diabetic patients. Ann N Y Acad Sci 1150:290-296

18. •- Gordin D, Forsblom C, Ronnback M et al (2008) Acute hyperglycaemia induces an inflammatory response in young patients with type 1 diabetes. Ann Med 40:627-633. This study compares the inflammatory response to hyperglycaemia in type 1 diabetic patients with that in controls

19. Miller AH, Maletic V, Raison CL (2009) Inflammation and its discontents: the role of cytokines in the pathophysiology of major depression. Biol Psychiatry 65:732-741

20. Ford DE, Erlinger TP (2004) Depression and C-reactive protein in US adults: data from the Third National Health and Nutrition Examination Survey. Arch Intern Med 164:1010-1014

21. Kiecolt-Glaser JK, McGuire L, Robles TF, Glaser R (2002) Emotions, morbidity, and mortality: new perspectives from psychoneuroimmunology. Annu Rev Psychol 53:83-107

22. Maes M (1999) Major depression and activation of the inflammatory response system. Adv Exp Med Biol 461:25-46

23. Cyranowski JM, Marsland AL, Bromberger JT, Whiteside TL, Chang Y, Matthews KA (2007) Depressive symptoms and production of proinflammatory cytokines by peripheral blood mononuclear cells stimulated in vitro. Brain Behav Immun 21:229-237

24. Raison CL, Capuron L, Miller AH (2006) Cytokines sing the blues: inflammation and the pathogenesis of depression. Trends Immunol 27:24-31

25. Sapolsky R, Rivier C, Yamamoto G, Plotsky P, Vale W (1987) Interleukin-1 stimulates the secretion of hypothalamic corticotropinreleasing factor. Science 238:522-524

26. Empana JP, Sykes DH, Luc G et al (2005) Contributions of depressive mood and circulating inflammatory markers to coronary heart disease in healthy European men: the Prospective Epidemiological Study of Myocardial Infarction (PRIME). Circulation 111:2299-2305

27. Lesperance F, Frasure-Smith N, Theroux P, Irwin M (2004) The association between major depression and levels of soluble intercellular adhesion molecule 1, interleukin-6, and C-reactive protein in patients with recent acute coronary syndromes. Am J Psychiatry 161:271-277

28. Crowe FL, Skeaff CM, Green TJ, Gray AR (2007) Serum phospholipid $n-3$ long-chain polyunsaturated fatty acids and physical and mental health in a population-based survey of New Zealand adolescents and adults. Am J Clin Nutr 86:12781285

29. Mamalakis G, Kiriakakis M, Tsibinos G et al (2008) Lack of an association of depression with n-3 polyunsaturated fatty acids in adipose tissue and serum phospholipids in healthy adults. Pharmacol Biochem Behav 89:6-10

30. Bot M, Pouwer F, Assies J et al (2010) Eicosapentaenoic acid as an add-on to antidepressant medication for co-morbid major depression in patients with diabetes mellitus: a randomized, double-blind placebo-controlled study. J Affect Disord 126:282286

31. Carney RM, Freedland KE, Rubin EH, Rich MW, Steinmeyer BC, Harris WS (2009) Omega-3 augmentation of sertraline in treatment of depression in patients with coronary heart disease: a randomized controlled trial. JAMA 302:1651-1657

32. Miller MR, Yin X, Seifert J et al (2011) Erythrocyte membrane omega-3 fatty acid levels and omega-3 fatty acid intake are not associated with conversion to type 1 diabetes in children with islet autoimmunity: The Diabetes Autoimmunity Study in the Young (DAISY). Pediatr Diabetes. doi:10.1111/ j.1399-5448.2011.00760.x

33. Zipitis CS, Akobeng AK (2008) Vitamin D supplementation in early childhood and risk of type 1 diabetes: a systematic review and meta-analysis. Arch Dis Child 93:512-517

34. Pittas AG, Dawson-Hughes B, Li T et al (2006) Vitamin D and calcium intake in relation to type 2 diabetes in women. Diabetes Care 29:650-656

35. Jorde R, Sneve M, Figenschau Y, Svartberg J, Waterloo K (2008) Effects of vitamin D supplementation on symptoms of depression in overweight and obese subjects: randomized double blind trial. J Intern Med 264:599-609

36. Howland RH (2011) Vitamin D and depression. J Psychosoc Nurs Ment Health Serv 49:15-18

37. Thacher TD, Clarke BL (2011) Vitamin D insufficiency. Mayo Clin Proc 86:50-60

38. Elhadd TA, Kennedy G, Hill A et al (1999) Abnormal markers of endothelial cell activation and oxidative stress in children, adolescents and young adults with type 1 diabetes with no clinical vascular disease. Diabetes Metab Res Rev 15:405-411

39. Evans JL, Goldfine ID, Maddux BA, Grodsky GM (2003) Are oxidative stress-activated signaling pathways mediators of insulin resistance and beta-cell dysfunction? Diabetes 52:1-8

40. Irie M, Asami S, Ikeda M, Kasai H (2003) Depressive state relates to female oxidative DNA damage via neutrophil activation. Biochem Biophys Res Commun 311:1014-1018

41. Collino M, Aragno M, Mastrocola R et al (2006) Modulation of the oxidative stress and inflammatory response by PPAR- $\gamma$ agonists in the hippocampus of rats exposed to cerebral ischemia/reperfusion. Eur J Pharmacol 530:70-80

42. Tiedge M, Lortz S, Drinkgern J, Lenzen S (1997) Relation between antioxidant enzyme gene expression and antioxidative defense status of insulin-producing cells. Diabetes 46:1733-1742

43. Halliwell B (1994) Free radicals, antioxidants, and human disease: curiosity, cause, or consequence? Lancet 344:721-724

44. - Martin-Gallan P, Carrascosa A, Gussinye M, Dominguez C (2007) Oxidative stress in childhood type 1 diabetes: results from a study covering the first 20 years of evolution. Free Radic Res 41:919-928. This clinical study examines markers of oxidative stress in childhood and adolescent onset of type 1 diabetes

45. Muruganandam A, Drouillard C, Thibert RJ, Cheung RM, Draisey TF, Mutus B (1992) Glutathione metabolic enzyme activities in diabetic platelets as a function of glycemic control. Thromb Res 67:385-397

46. Mylona-Karayanni C, Gourgiotis D, Bossios A, Kamper EF (2006) Oxidative stress and adhesion molecules in children with type 1 diabetes mellitus: a possible link. Pediatr Diabetes 7:5159

47. Hong JH, Kim MJ, Park MR et al (2004) Effects of vitamin E on oxidative stress and membrane fluidity in brain of streptozotocininduced diabetic rats. Clin Chim Acta 340:107-115 
48. Kamboj SS, Chopra K, Sandhir R (2008) Neuroprotective effect of $\mathrm{N}$-acetylcysteine in the development of diabetic encephalopathy in streptozotocin-induced diabetes. Metab Brain Dis 23:427-443

49. Ates O, Cayli SR, Yucel N et al (2007) Central nervous system protection by resveratrol in streptozotocin-induced diabetic rats. J Clin Neurosci 14:256-260

50. Tomlinson DR, Gardiner NJ (2008) Glucose neurotoxicity. Nat Rev Neurosci 9:36-45

51. Evans JL, Goldfine ID, Maddux BA, Grodsky GM (2002) Oxidative stress and stress-activated signaling pathways: a unifying hypothesis of type 2 diabetes. Endocr Rev 23:599622

52. Kuhad A, Chopra K (2007) Curcumin attenuates diabetic encephalopathy in rats: behavioral and biochemical evidences. Eur J Pharmacol 576:34-42

53. Pfeffer KD, Huecksteadt TP, Hoidal JR (1994) Xanthine dehydrogenase and xanthine oxidase activity and gene expression in renal epithelial cells. Cytokine and steroid regulation. J Immunol 153:1789-1797

54. Khanzode SD, Dakhale GN, Khanzode SS, Saoji A, Palasodkar R (2003) Oxidative damage and major depression: the potential antioxidant action of selective serotonin re-uptake inhibitors. Redox Rep 8:365-370

55. Maes M, de Vos N, Pioli R et al (2000) Lower serum vitamin E concentrations in major depression. Another marker of lowered antioxidant defenses in that illness. J Affect Disord 58:241-246

56. Sarandol A, Sarandol E, Eker SS, Erdinc S, Vatansever E, Kirli S (2007) Major depressive disorder is accompanied with oxidative stress: short-term antidepressant treatment does not alter oxidative-antioxidative systems. Hum Psychopharmacol 22:6773

57. Michel TM, Frangou S, Thiemeyer D et al (2007) Evidence for oxidative stress in the frontal cortex in patients with recurrent depressive disorder - a postmortem study. Psychiatry Res 151:145-150

58. You JM, Yun SJ, Nam KN, Kang C, Won R, Lee EH (2009) Mechanism of glucocorticoid-induced oxidative stress in rat hippocampal slice cultures. Can J Physiol Pharmacol 87:440-447

59. Bedard K, Krause KH (2007) The NOX family of ROSgenerating NADPH oxidases: physiology and pathophysiology. Physiol Rev 87:245-313

60. Eren I, Naziroglu M, Demirdas A et al (2007) Venlafaxine modulates depression-induced oxidative stress in brain and medulla of rat. Neurochem Res 32:497-505

61. Rezin GT, Amboni G, Zugno AI, Quevedo J, Streck EL (2009) Mitochondrial dysfunction and psychiatric disorders. Neurochem Res 34:1021-1029

62. da Silva HA, Sitta A, Barschak AG et al (2007) Oxidative stress parameters in diabetic rats submitted to forced swimming test: the clonazepam effect. Brain Res 1154:137-143

63. Gomez R, Barros HM (2000) Ethopharmacology of the antidepressant effect of clonazepam in diabetic rats. Pharmacol Biochem Behav 66:329-335

64. Porsolt RD, Le Pichon M, Jalfre M (1977) Depression: a new animal model sensitive to antidepressant treatments. Nature 266:730-732

65. Moretti A, Gorini A, Villa RF (2003) Affective disorders, antidepressant drugs and brain metabolism. Mol Psychiatry 8:773-785

66. Craft S, Watson GS (2004) Insulin and neurodegenerative disease: shared and specific mechanisms. Lancet Neurol 3:169178

67. Huang CC, Lee CC, Hsu KS (2010) The role of insulin receptor signaling in synaptic plasticity and cognitive function. Chang Gung Med J 33:115-125
68. Reger MA, Watson GS, Green PS et al (2008) Intranasal insulin improves cognition and modulates $\beta$-amyloid in early $\mathrm{AD}$. Neurology 70:440-448

69. Crandall EA, Gillis MA, Fernstrom JD (1981) Reduction in brain serotonin synthesis rate in streptozotocin-diabetic rats. Endocrinology 109:310-312

70. Fernstrom JD (2005) Branched-chain amino acids and brain function. J Nutr 135:1539S-1546S

71. Crandall EA, Fernstrom JD (1983) Effect of experimental diabetes on the levels of aromatic and branched-chain amino acids in rat blood and brain. Diabetes 32:222-230

72. Herrera R, Manjarrez G, Nishimura E, Hernandez J (2003) Serotonin-related tryptophan in children with insulin-dependent diabetes. Pediatr Neurol 28:20-23

73. Castellino P, Luzi L, Simonson DC, Haymond M, DeFronzo RA (1987) Effect of insulin and plasma amino acid concentrations on leucine metabolism in man. Role of substrate availability on estimates of whole body protein synthesis. J Clin Invest 80:1784-1793

74. Meek SE, Persson M, Ford GC, Nair KS (1998) Differential regulation of amino acid exchange and protein dynamics across splanchnic and skeletal muscle beds by insulin in healthy human subjects. Diabetes 47:1824-1835

75. Inchiostro S, Biolo G, Bruttomesso D et al (1992) Effects of insulin and amino acid infusion on leucine and phenylalanine kinetics in type 1 diabetes. Am J Physiol 262:E203-E210

76. Manjarrez G, Herrera R, Leon M, Hernandez RJ (2006) A low brain serotonergic neurotransmission in children with type 1 diabetes detected through the intensity dependence of auditoryevoked potentials. Diabetes Care 29:73-77

77. • Crandall EA, Fernstrom JD (1980) Acute changes in brain tryptophan and serotonin after carbohydrate or protein ingestion by diabetic rats. Diabetes 29: 460-466. This study investigates insulin-induced effects on brain levels of tryptophan in streptozotocin-treated rats

78. - Rosenthal JM, Amiel SA, Yaguez L, et al. (2001) The effect of acute hypoglycemia on brain function and activation: a functional magnetic resonance imaging study. Diabetes 50:16181626. This study shows the effect of hypoglycaemia upon the activation of human brain regions

79. Hershey T, Perantie DC, Warren SL, Zimmerman EC, Sadler M, White NH (2005) Frequency and timing of severe hypoglycemia affects spatial memory in children with type 1 diabetes. Diabetes Care 28:2372-2377

80. Jacobson AM, Musen G, Ryan CM et al (2007) Long-term effect of diabetes and its treatment on cognitive function. N Engl J Med 356:1842-1852

81. Ryan CM (1988) Neurobehavioral complications of type I diabetes. Examination of possible risk factors. Diabetes Care 11:86-93

82. Kaufman FR, Epport K, Engilman R, Halvorson M (1999) Neurocognitive functioning in children diagnosed with diabetes before age 10 years. J Diabetes Complications 13:31-38

83. Strachan MW, Deary IJ, Ewing FM, Frier BM (2000) Recovery of cognitive function and mood after severe hypoglycemia in adults with insulin-treated diabetes. Diabetes Care 23:305-312

84. Lebinger TG, Saenger P, Fukushima DK, Kream J, Wu R, Finkelstein JW (1983) Twenty-four-hour cortisol profiles demonstrate exaggerated nocturnal rise in diabetic children. Diabetes Care 6:506-509

85. - Roy MS, Roy A, Gallucci WT et al (1993) The ovine corticotropin-releasing hormone-stimulation test in type I diabetic patients and controls: suggestion of mild chronic hypercortisolism. Metabolism 42:696-700. This study examines HPA-axis hyperactivity in patients with type 1 diabetes 
86. Hudson JI, Hudson MS, Rothschild AJ, Vignati L, Schatzberg AF, Melby JC (1984) Abnormal results of dexamethasone suppression tests in nondepressed patients with diabetes mellitus. Arch Gen Psychiatry 41:1086-1089

87. •- Chan O, Inouye K, Riddell MC, Vranic M, Matthews SG (2003) Diabetes and the hypothalamo-pituitary-adrenal (HPA) axis. Minerva Endocrinol 28:87-102. This review addresses the mechanism of HPA axis hyperactivation in diabetes

88. Gibbons J, McHugh P (1962) Plasma cortisol in depressive illness. J Psychiat Res 1:162-171

89. Nemeroff CB, Krishnan KR, Reed D, Leder R, Beam C, Dunnick NR (1992) Adrenal gland enlargement in major depression. A computed tomographic study. Arch Gen Psychiatry 49:384-387

90. Nemeroff CB, Widerlov E, Bissette G et al (1984) Elevated concentrations of CSF corticotropin-releasing factor-like immunoreactivity in depressed patients. Science 226:1342-1344

91. Ribeiro SC, Tandon R, Grunhaus L, Greden JF (1993) The DST as a predictor of outcome in depression: a meta-analysis. Am J Psychiatry 150:1618-1629

92. Starkman MN, Schteingart DE, Schork MA (1981) Depressed mood and other psychiatric manifestations of Cushing's syndrome: relationship to hormone levels. Psychosom Med 43:3-18

93. Wolkowitz OM, Reus VI (1999) Treatment of depression with antiglucocorticoid drugs. Psychosom Med 61:698-711

94. - Chan O, Chan S, Inouye K, Vranic M, Matthews SG (2001) Molecular regulation of the hypothalamo-pituitary-adrenal axis in streptozotocin-induced diabetes: effects of insulin treatment. Endocrinology 142:4872-4879. This study reports the effect of insulin treatment on HPA axis activity

95. McEwen BS, de Kloet ER, Rostene W (1986) Adrenal steroid receptors and actions in the nervous system. Physiol Rev 66:1121-1188

96. Steckler T, Holsboer F, Reul JM (1999) Glucocorticoids and depression. Baillieres Best Pract Res Clin Endocrinol Metab 13:597-614

97. Gillespie CF, Nemeroff CB (2005) Hypercortisolemia and depression. Psychosom Med 67(Suppl 1):S26-28

98. Modell S, Lauer CJ, Schreiber W, Huber J, Krieg JC, Holsboer F (1998) Hormonal response pattern in the combined DEX-CRH test is stable over time in subjects at high familial risk for affective disorders. Neuropsychopharmacology 18:253-262

99. Chiodini I, Adda G, Scillitani A et al (2007) Cortisol secretion in patients with type 2 diabetes: relationship with chronic complications. Diabetes Care 30:83-88

100. Brands AM, Biessels GJ, de Haan EH, Kappelle LJ, Kessels RP (2005) The effects of type 1 diabetes on cognitive performance: a meta-analysis. Diabetes Care 28:726-735

101. American Psychiatric Association (1994) Diagnostic and statistical manual of mental disorders, 4th edn. American Psychiatric Association, Washington, DC

102. McEwen BS, Magarinos AM, Reagan LP (2002) Studies of hormone action in the hippocampal formation: possible relevance to depression and diabetes. J Psychosom Res 53:883-890

103. - Reagan LP, Magarinos AM, McEwen BS (1999) Neurological changes induced by stress in streptozotocin diabetic rats. Ann N Y Acad Sci 893:126-137. This study identifies the hippocampus as a vulnerable organ in STZ-treated diabetes

104. Ferguson SC, Blane A, Wardlaw J et al (2005) Influence of an early-onset age of type 1 diabetes on cerebral structure and cognitive function. Diabetes Care 28:1431-1437
105. Ho MS, Weller NJ, Ives FJ et al (2008) Prevalence of structural central nervous system abnormalities in early-onset type 1 diabetes mellitus. J Pediatr 153:385-390

106. Lobnig BM, Kromeke O, Optenhostert-Porst C, Wolf OT (2006) Hippocampal volume and cognitive performance in longstanding type 1 diabetic patients without macrovascular complications. Diabet Med 23:32-39

107. Lunetta M, Damanti AR, Fabbri G, Lombardo M, Di Mauro M, Mughini L (1994) Evidence by magnetic resonance imaging of cerebral alterations of atrophy type in young insulin-dependent diabetic patients. J Endocrinol Invest 17:241-245

108. van Harten B, de Leeuw FE, Weinstein HC, Scheltens P, Biessels GJ (2006) Brain imaging in patients with diabetes: a systematic review. Diabetes Care 29:2539-2548

109. - Nestler EJ, Barrot M, DiLeone RJ, Eisch AJ, Gold SJ, Monteggia LM (2002) Neurobiology of depression. Neuron 34:13-25. This review examines the neural circuitry and HPA axis dysfunction in $M D D$

110. •• Sheline YI, Wang PW, Gado MH, Csernansky JG, Vannier MW (1996) Hippocampal atrophy in recurrent major depression. Proc Natl Acad Sci USA 93:3908-3913. This study describes the structural hippocampal changes in patients with $M D D$

111. Heim C, Nemeroff CB (2001) The role of childhood trauma in the neurobiology of mood and anxiety disorders: preclinical and clinical studies. Biol Psychiatry 49:1023-1039

112. Lloyd AJ, Ferrier IN, Barber R, Gholkar A, Young AH, O’Brien JT (2004) Hippocampal volume change in depression: late- and early-onset illness compared. Br J Psychiatry 184:488-495

113. Reiche EM, Morimoto HK, Nunes SM (2005) Stress and depression-induced immune dysfunction: implications for the development and progression of cancer. Int Rev Psychiatry 17:515-527

114. Chen B, Dowlatshahi D, MacQueen GM, Wang JF, Young LT (2001) Increased hippocampal BDNF immunoreactivity in subjects treated with antidepressant medication. Biol Psychiatry 50:260-265

115. Brunoni AR, Lopes M, Fregni F (2008) A systematic review and meta-analysis of clinical studies on major depression and BDNF levels: implications for the role of neuroplasticity in depression. Int J Neuropsychopharmacol 11:1169-1180

116. Krishnan V, Nestler EJ (2010) Linking molecules to mood: new insight into the biology of depression. Am J Psychiatry 167:1305-1320

117. Chaldakov GN, Tonchev AB, Manni L et al (2007) Comment on: Krabbe KS, Nielsen AR, Krogh-Madsen R et al (2007) Brainderived neurotrophic factor (BDNF) and type 2 diabetes. Diabetologia 50:431-438. Diabetologia 50:1781-1782

118. Krabbe KS, Nielsen AR, Krogh-Madsen R et al (2007) Brainderived neurotrophic factor (BDNF) and type 2 diabetes. Diabetologia 50:431-438

119. Yamanaka M, Itakura Y, Inoue T et al (2006) Protective effect of brain-derived neurotrophic factor on pancreatic islets in obese diabetic mice. Metabolism 55:1286-1292

120. Wichers M, Kenis G, Jacobs N et al (2008) The BDNF Val ${ }^{66}$ Met $\times 5$-HTTLPR $\times$ child adversity interaction and depressive symptoms: an attempt at replication. Am J Med Genet B Neuropsychiatr Genet 147B:120-123

121. Elzinga BM, Molendijk ML, Oude Voshaar RC et al (2011) The impact of childhood abuse and recent stress on serum brainderived neurotrophic factor and the moderating role of BDNF Val66Met. Psychopharmacology (Berl) 214:319-328 